\title{
Coherently Controlled Asymmetric Synthesis with Achiral Light
}

\author{
Moshe Shapiro and Einat Frishman \\ Chemical Physics Department, The Weizmann Institute of Science, Rehovot, Israel 76100 \\ Paul Brumer \\ Chemical Physics Theory Group, Department of Chemistry, University of Toronto, \\ Toronto, Canada M5S $3 \mathrm{H} 6$
}

(Received 10 June 1999)

\begin{abstract}
A laser-based method of increasing the enantiomeric excess of a chiral enantiomer in a racemic mixture is described. Neither the initial reagents nor the incident light need be chiral. Both formal and computational results show that enhancement of the enantiomer of choice, controlled by laser parameters, can be extensive.

PACS numbers: $31.10 .+\mathrm{z}, 33.80 .-\mathrm{b}$
\end{abstract}

The existence of enantiomers, i.e., pairs of chiral molecules related to one another through inversion $I$, is one of the fundamental broken symmetries in nature [1]. It is also one of great practical importance because biological processes are often stereospecific, motivating a long-standing interest in asymmetric synthesis, i.e., molecular processes which preferentially produce one of the enantiomeric pairs.

It is a long-standing belief that asymmetric synthesis must necessary involve either reactants or reaction conditions which are chiral [2], i.e., display a decided handedness. Thus, for example, previous efforts to use light-matter interactions to alter the enantiomeric excess in a racemic mixture used either circular or elliptically polarized light [3]. Here we show that preferential production of a chiral molecule in a molecular process can occur even though neither the system Hamiltonian nor the initial conditions are chiral. In particular, we show that one can design achiral laser scenarios which encode quantum coherences in the molecule to selectively enhance the production of either the right- (denoted $D$ ) or left- (denoted $L$ ) handed enantiomer, even when starting with a racemic mixture.

To do so we consider a molecule with two enantiomers $L$ and $D$, with a common excited electronic state whose potential surface, denoted $G$, is achiral [4]. This excited electronic state has stationary rovibrational states which are either symmetric or antisymmetric with respect to $I$. The scenario that we advocate, which is a significant extension of the coherent control approach [6,7], is described below. In particular, a racemic mixture of $L$ and $D$ is irradiated with a well-defined sequence of achiral coherent laser pulses to form a specified coherent superposition of rovibrational states of $G$. This excited system then returns to the ground electronic state by radiative emission. By successively irradiating the system and allowing radiative emission and collisional relaxation, we show that one can enhance the concentration of either enantiomer $L$ or $D$ by varying the laser pulse character- istics. We call this scenario the "laser distillation of chiral enantiomers."

Consider then the time dependent Schrödinger equation for a molecule with Hamiltonian $H_{M}$ in the presence of a series of laser pulses. In general we may deal with lasers which are not fully coherent but for simplicity we focus here on transform limited pulses of linearly polarized light. The electric field, here comprised of two pulses, is given by $\mathbf{E}(t)=\sum_{k=0,1} \mathbf{E}_{k}(t) \equiv \sum_{k=0,1} 2 \hat{\boldsymbol{\epsilon}}_{k} \operatorname{Re}\left[\boldsymbol{\varepsilon}_{k}(t) \exp \left(i \omega_{k} t\right)\right]$, where $\varepsilon_{k}(t)$ is the pulse envelope, $\omega_{k}$ is the central laser frequency, and $\hat{\epsilon}_{k}$ is the polarization direction. The time dependent Schrödinger equation is $i \hbar \partial|\Psi\rangle / \partial t=H_{\text {tot }}(t)|\Psi\rangle$ with

$$
H_{\mathrm{tot}}(t)=H_{M}-\sum_{k} \vec{\mu}_{k} \cdot \mathbf{E}_{k}(t)
$$

and $\vec{\mu}_{k}$ is the transition-dipole moment for the electronic transition induced by $\mathbf{E}_{k}(t)$. Expanding $|\Psi\rangle$ in eigenstates $|j\rangle$ of the molecular Hamiltonian [i.e., $H_{M}|j\rangle=E_{j}|j\rangle$ ] as $|\Psi\rangle=\sum_{j} b_{j} \exp \left(-i E_{j} t / \hbar\right)|j\rangle$ and substituting into the Schrödinger equation gives the standard set of coupled equations:

$$
\dot{b}_{i}=\frac{i}{\hbar} \sum_{j k} b_{j} \exp \left(-i \omega_{j i} t\right)\left\langle i\left|\vec{\mu}_{k} \cdot \mathbf{E}_{k}(t)\right| j\right\rangle
$$

where $\omega_{j i}=\left(E_{j}-E_{i}\right) / \hbar$.

As an example of an effective control scenario, consider the $D$ or $L$ enantiomer in its ground electronic states with $|D\rangle$ and $|L\rangle$ denoting vibrotational eigenstates of energy $E_{D}=E_{L}$. We choose $\varepsilon_{1}(t)$ so as to excite the system to two eigenstates $|1\rangle$ and $|2\rangle$, of energies $E_{1}$ and $E_{2}$, of the electronically excited $G$. The states $|1\rangle$ and $|2\rangle$ are coupled by an additional laser field (see Fig. 1) $\varepsilon_{0}(t)$. Specifically, we choose $\mathbf{E}(t)$ to be composed of two linearly polarized light pulses, with $\omega_{0}$ in near resonance with $\omega_{21} \equiv\left(E_{2}-E_{1}\right) / \hbar$, and $\omega_{1}$ chosen to lie 


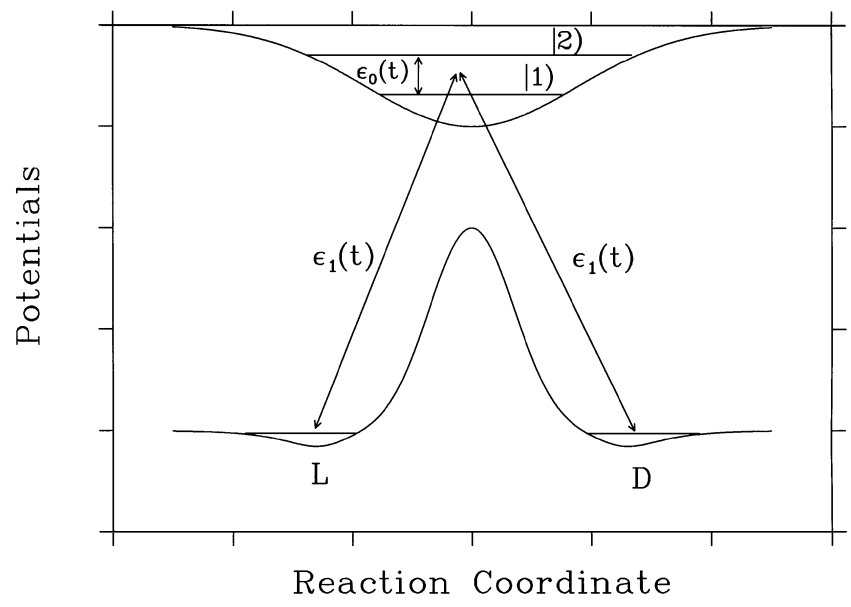

FIG. 1. Sample control scenario.

between $\omega_{1 D} \equiv\left(E_{1}-E_{D}\right) / \hbar$, and $\omega_{2 D} \equiv\left(E_{2}-E_{D}\right) / \hbar$ (see Fig. 1). In this case only four molecular states are relevant and $|\Psi\rangle$ is expanded as

$$
\begin{aligned}
|\Psi\rangle= & b_{D}(t) \exp \left(-i E_{D} t / \hbar\right)|D\rangle+b_{L}(t) \exp \left(-i E_{L} t / \hbar\right)|L\rangle \\
& +b_{1}(t) \exp \left(-i E_{1} t / \hbar\right)|1\rangle+b_{2}(t) \exp \left(-i E_{2} t / \hbar\right)|2\rangle .
\end{aligned}
$$

Equation (2), in the rotating wave approximation, is then given by

$$
\begin{aligned}
\dot{b}_{1}= & i \exp \left(i \Delta_{1} t\right)\left[\Omega_{D, 1}^{*} b_{D}+\Omega_{L, 1}^{*} b_{L}\right] \\
& +i \exp \left(i \Delta^{(2)} t\right) \Omega_{0}^{*} b_{2} \\
\dot{b}_{2}= & i \exp \left(i \Delta_{2} t\right)\left[\Omega_{D, 2}^{*} b_{D}+\Omega_{L, 2}^{*} b_{L}\right] \\
& +i \exp \left(-i \Delta^{(2)} t\right) \Omega_{0} b_{1} \\
\dot{b}_{D}= & i \exp \left(-i \Delta_{1} t\right) \Omega_{D, 1} b_{1}+i \exp \left(-i \Delta_{2} t\right) \Omega_{D, 2} b_{2}, \\
\dot{b}_{L}= & i \exp \left(-i \Delta_{1} t\right) \Omega_{L, 1} b_{1}+i \exp \left(-i \Delta_{2} t\right) \Omega_{L, 2} b_{2}
\end{aligned}
$$

where $\Omega_{i j}(t) \equiv \mu_{i j}^{(1)} \varepsilon_{1}(t) / \hbar, \quad \Omega_{0} \equiv \mu_{21}^{(0)} \varepsilon_{0}(t) / \hbar, \quad \Delta_{j} \equiv$ $\omega_{j D}-\omega_{1}, \quad \Delta^{(2)} \equiv \omega_{21}-\omega_{0}, \quad$ where $\quad \mu_{i j}^{(k)} \equiv$ $\left\langle i\left|\vec{\mu}_{k} \cdot \hat{\epsilon}_{k}\right| j\right\rangle$, with $i=D, L ; k=0,1$ and $j=1,2$. Note, to avoid confusion, that all computations carried out below assume that the initial state is either $|D\rangle$ [i.e., $b_{L}(0)=0$ ] or $|L\rangle$ [i.e., $b_{D}(0)=0$ ]. No initial superposition of $|D\rangle$ and $|L\rangle$ is assumed.

The essence of the laser distillation process lies in choosing the laser of central frequency $\omega_{1}$ so that it excites the system to a state $|1\rangle$ which is symmetric with respect to the inversion operation $I$, and to a state $|2\rangle$ which is antisymmetric with respect to $I$. By contrast, $|D\rangle$ and $|L\rangle$ do not share these symmetries but are related to one another through inversion (i.e., $I|D\rangle=-|L\rangle, I|L\rangle=-|D\rangle$ whereas $I|1\rangle=|1\rangle, I|2\rangle=-|2\rangle)$. Hence, $\varepsilon_{1}(t)$ is chosen unimodal if $|1\rangle$ and $|2\rangle$ are adjacent levels, or bimodal if the pulse needs to be shaped to predominantly excite states of the desired symmetries. Note that $|D\rangle$ and $|L\rangle$ are not eigenstates of the parity operator or of $H_{M}$. Hence there are no parity constraints $[5,8]$ preventing either the dipole excitation of both $|1\rangle$ and $|2\rangle$ from $|D\rangle$ and $|L\rangle$, or dipole transitions between $|1\rangle$ and $|2\rangle$.

To consider the nature of the Rabi frequencies $\Omega$ in Eq. (4) we rewrite $|D\rangle$ and $|L\rangle$ in terms of a symmetric state $|S\rangle=[|D\rangle-|L\rangle] / 2$ and an antisymmetric state $|A\rangle=[|D\rangle+|L\rangle] / 2$. The relevant matrix elements are then of the form:

$$
\begin{aligned}
\left\langle 1\left|\mu^{(1)}\right| D\right\rangle & =\left\langle 1\left|\mu^{(1)}\right| A+S\right\rangle=\left\langle 1\left|\mu^{(1)}\right| A\right\rangle, \\
\left\langle 1\left|\mu^{(1)}\right| L\right\rangle & =\left\langle 1\left|\mu^{(1)}\right| A-S\right\rangle=\left\langle 1\left|\mu^{(1)}\right| A\right\rangle, \\
\left\langle 2\left|\mu^{(1)}\right| D\right\rangle & =\left\langle 2\left|\mu^{(1)}\right| A+S\right\rangle=\left\langle 2\left|\mu^{(1)}\right| S\right\rangle, \\
\left\langle 2\left|\mu^{(1)}\right| L\right\rangle & =\left\langle 2\left|\mu^{(1)}\right| A-S\right\rangle=-\left\langle 2\left|\mu^{(1)}\right| S\right\rangle .
\end{aligned}
$$

That is, $\Omega_{D, 1}=\Omega_{L, 1}, \Omega_{D, 2}=-\Omega_{L, 2}$, and Eq. (4) therefore becomes

$$
\begin{aligned}
& \dot{b}_{1}=i \exp \left(i \Delta_{1} t\right) \Omega_{D, 1}^{*}\left[b_{D}+b_{L}\right]+i \exp \left(i \Delta^{(2)} t\right) \Omega_{0}^{*} b_{2}, \\
& \dot{b}_{2}=i \exp \left(i \Delta_{2} t\right) \Omega_{D, 2}^{*}\left[b_{D}-b_{L}\right]+i \exp \left(-i \Delta^{(2)} t\right) \Omega_{0} b_{1} \\
& \dot{b}_{D}=i \exp \left(-i \Delta_{1} t\right) \Omega_{D, 1} b_{1}+i \exp \left(-i \Delta_{2} t\right) \Omega_{D, 2} b_{2} \\
& \dot{b}_{L}=i \exp \left(-i \Delta_{1} t\right) \Omega_{D, 1} b_{1}-i \exp \left(-i \Delta_{2} t\right) \Omega_{D, 2} b_{2}
\end{aligned}
$$

The essence of optically controlled enantioselectivity lies in the relationship $\Omega_{D, 2}=-\Omega_{L, 2}$ and its effect on the dynamical equations for the level populations [Eq. (6)]. Note specifically that the equation for $\dot{b}_{D}(t)$ is different than the equation for $\dot{b}_{L}(t)$, due to the sign difference in the last term to Eq. (6). Although not sufficient to ensure enantiomeric selectivity, the ultimate consequence of this difference is that populations of $|D\rangle$ and $|L\rangle$ after laser excitation are different in the presence of radiative coupling between levels $|1\rangle$ and $|2\rangle$.

To obtain quantitative estimates for the extent of obtainable control we have numerically solved Eq. (4) for model cases assuming Gaussian pulses: $\varepsilon_{\ell}(t)=$ $\beta_{\ell} \exp \left\{-\left[\left(t-t_{\ell}\right) / \alpha_{\ell}\right]^{2}\right\} \quad(\ell=0,1)$ and system parameters $\left\langle 1\left|\mu^{(1)}\right| D\right\rangle=\left\langle 1\left|\mu^{(1)}\right| L\right\rangle=\left\langle 2\left|\mu^{(1)}\right| L\right\rangle=$ $-\left\langle 2\left|\mu^{(1)}\right| D\right\rangle=1$ a.u., $\quad\left\langle 1\left|\mu^{(0)}\right| 2\right\rangle=1$ a.u., $\quad \omega_{2,1}=$ $100 \mathrm{~cm}^{-1}$, and $\Delta^{(2)}=0$. Figure 2 displays the final probabilities $P_{D}=\left|b_{D}(\infty)\right|^{2}, P_{L}=\left|b_{L}(\infty)\right|^{2}$ in $|D\rangle$ and $|L\rangle$, after a single pulse sequence, for a variety of pulse parameters. Results are shown for various values of $\Delta_{1}$ at various different pulse powers assuming that one starts solely with $D$, solely with $L$, or with a racemic mixture of both enantiomers. Clearly, the responses of $D$ and $L$ to the pulses are quite different and, for particular parameters, one can significantly enhance the population of one chiral enantiomer over the other. For example, for $\Delta_{1}=-125 \mathrm{~cm}^{-1}, \beta_{0}=\beta_{1}=1.5 \times 10^{-3}$, a racemic mixture of $D$ and $L$ can be converted, after a single pulse, to an enantiomerically enriched mixture with predominantly $D$. Tuning to $\Delta_{1}=25 \mathrm{~cm}^{-1}$ at the same power results in a significant enhancement of $L$.

Figure 2, however, only provides input into a computation of the overall result. In the overall process we begin 


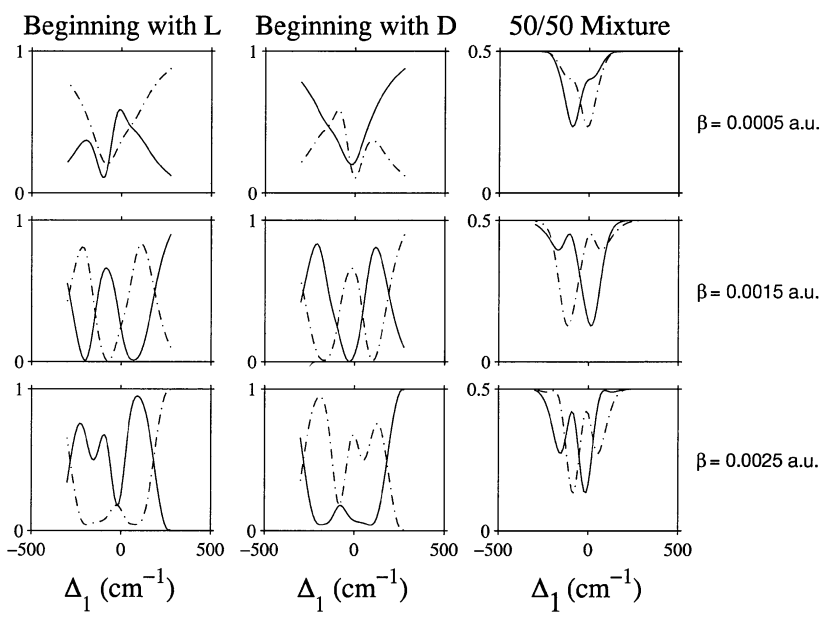

FIG. 2. Probabilities of populating the $|D\rangle$ (solid lines) and $|L\rangle$ (dot-dashed lines) after laser excitation, but prior to relaxation, as a function of $\Delta_{1}$. Three different cases are shown, corresponding to three different initial conditions: (1) only state $|L\rangle$ occupied, (2) only state $|D\rangle$ occupied, and (3) both $|D\rangle$ and $|L\rangle$ equally occupied. Results are shown for three different laser powers $\left(\beta \equiv \beta_{0}=\beta_{1}\right)$, where Gaussian pulses are assumed with $\alpha_{0}=$ $\alpha_{1}=0.15 \mathrm{psec}$, and $t_{0}=t_{1}$.

with an incoherent mixture of $N_{D}$ molecules of type $D$ and $N_{L}$ molecules of type $L$. In the first step the system is excited, as above, with a laser pulse sequence. In the second step, the system collisionally and radiatively relaxes so that all the population returns to the ground state to produce an incoherent mixture of $|L\rangle$ and $|D\rangle$. This pair of steps is then repeated until the populations of $|L\rangle$ and $|D\rangle$ reach convergence.

To obtain the result computationally note that the population after laser excitation, but before radiative relaxation, consists of the weighted sum of the results of two computations: $N_{D}$ times the results of laser excitation starting solely with molecules in $|D\rangle$, plus $N_{L}$ times the results of laser excitation starting solely with molecules in $|L\rangle$. If $P_{D}$ and $P_{L}$ denote the probabilities of $|D\rangle$ and $|L\rangle$ resulting from laser excitation assuming the first of these initial conditions, and $P_{D}^{\prime}$ and $P_{L}^{\prime}$ for the results of excitation following from the second of these initial conditions, then the populations of $|D\rangle$ and $|L\rangle$ after laser excitation of the mixture are $N_{D} P_{D}+N_{L} P_{D}^{\prime}$ and $N_{D} P_{L}+N_{L} P_{L}^{\prime}$, respectively. The remainder of the population, $\quad N_{D}\left[1-P_{D}-P_{L}\right]+N_{L}\left[1-P_{D}^{\prime}-P_{L}^{\prime}\right]$, is in the upper two levels $|1\rangle$ and $|2\rangle$. Radiative emission from levels $|1\rangle$ and $|2\rangle$ then follows, with the excited population dividing itself equally between $|D\rangle$ and $|L\rangle$. The resultant populations $\mathcal{N}_{D}$ and $\mathcal{N}_{L}$ is ground state $|D\rangle$ and $|L\rangle$ are then $\mathcal{N}_{D}=$ $0.5 N_{D}\left[1+P_{D}-P_{L}\right]+0.5 N_{L}\left[1+P_{D}^{\prime}-P_{L}^{\prime}\right] \quad$ and $\mathcal{N}_{L}=N_{D}+N_{L}-\mathcal{N}_{D}$.

The sequence of laser excitation followed by collisional relaxation and radiative emission is then iterated to convergence, i.e., $\mathcal{N}_{D}=N_{D}$, and $\mathcal{N}_{L}=$ $N_{L}$. These conditions reduce to $N_{D}\left(1-P_{D}+P_{L}\right)=$ $N_{L}\left(1+P_{D}^{\prime}-P_{L}^{\prime}\right)$. If the total population is chosen to be normalized $\left(N_{D}+N_{L}=1\right)$, then the final probabilities $\mathcal{P}_{D}, \mathcal{P}_{L}$ of populating states $|D\rangle$ and $|L\rangle$ are

$$
\mathcal{P}_{D}=\frac{1+P_{D}^{\prime}-P_{L}^{\prime}}{2-P_{D}+P_{L}+P_{D}^{\prime}-P_{L}^{\prime}}
$$

with $\mathcal{P}_{L}=1-\mathcal{P}_{D}$.

Results for the converged probabilities for the cases depicted in Fig. 2 are shown in Fig. 3. The results clearly show substantially enhanced enantiomeric ratios at various choices of control parameters. For example, at $\beta \equiv \beta_{0}=$ $\beta_{1}=1.5 \times 10^{-3}$, tuning $\Delta_{1}$ to $50 \mathrm{~cm}^{-1}$ gives a preponderance of $L$ whereas tuning to the $\Delta_{1}=-125 \mathrm{~cm}^{-1}$ gives more $D$.

Numerous parameters in this system, such as the pulse shape, time delay between pulses, pulse frequencies, and pulse powers, etc., can be varied to affect the final $L$ to $D$ ratio. For example, Fig. 4 shows the dependence on the ratio of field strengths $\beta_{0} / \beta_{1}$ for various values of $\Delta t=t_{0}-t_{1}$. Changing these parameters is clearly seen to alter the $L$ to $D$ ratio.

Conditions on the structure of the molecule under which this method is applicable have been described above and such molecules are expected to exist [4]. However, in many cases an excited state $G$ with the required characteristics does not exist below the dissociation energy. In such cases one may be able to apply the laser distillation procedure by adding a molecule $B$ to the initial $L, D$

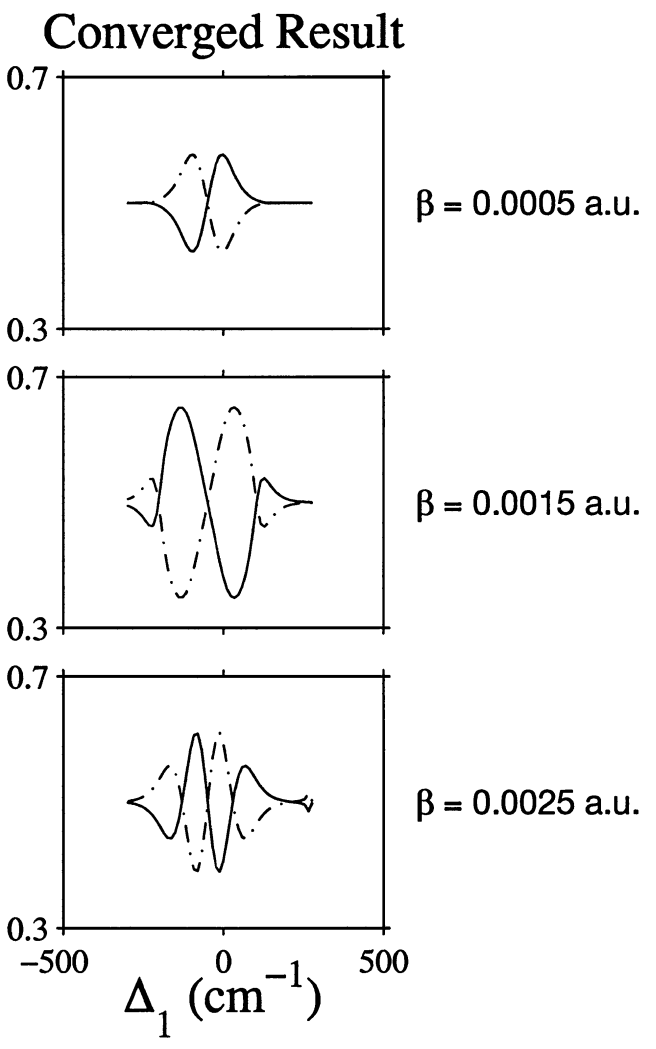

FIG. 3. Results for the cases in Fig. 2, but after a convergent series of steps comprised of radiative excitation, followed by collisional and radiative relaxation. 


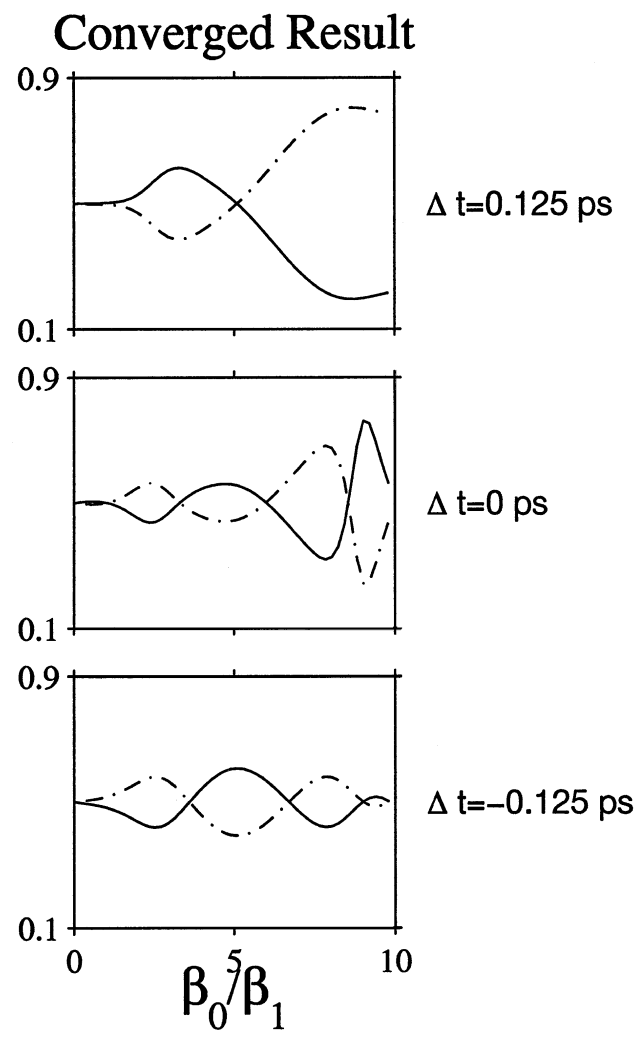

FIG. 4. Converged results as a function of field strength ratios with $\Delta_{1}=0, \alpha_{0}=\alpha_{1}=0.25$ psec, and $\beta_{1}=1.0 \times 10^{-4}$ a.u. for three values of $\Delta t$.

mixture to form weakly bound $L-B$ and $B-D$, which are themselves right- and left-handed enantiomeric pairs. The molecule $B$ is chosen so that electronic excitation of $B-D$ and $L-B$ forms an excited species $G$ which has stationary rovibrational states which are either symmetric or antisymmetric with respect to inversion through $I$. The species $L-B$ and $B-D$ now serve as the $L$ and $D$ enantiomers in the general scenario above and the laser distillation procedure described above then applies. Further, the molecule $B$ serves a catalyst that may be removed from the final product by traditional chemical means.

For example, $L$ and $D$ might be the left- and right-handed enantiomers of a chiral alcohol, and $B$ is the ketone derived from this alcohol (see Fig. 5). In this case, preliminary studies [9] of the electronic structure of the alcohol-ketone system show that there are weakly bound chiral alcohol-ketone minima in the ground electronic state, as desired; further work is in progress to determine the excited state structures [9].

In summary, we have shown that a chiral outcome, the enhancement of a particular enantiomer, can arise by coherently encoding quantum interference information in the excitation of a racemic mixture. The fact that the initial state displays a broken symmetry and that the excited state has states which are either symmetric or antisymmetric with respect to $I$ allows for the creation of a superposition

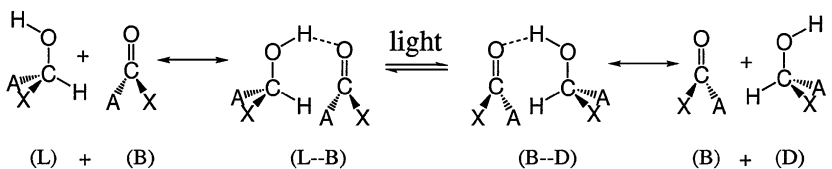<smiles>[Y]C1CC([X])([Y])OC[OH+]1</smiles>

(G)

FIG. 5. Sample scenario for enhanced enantiomeric selectivity in a racemic mixture of two chiral alcohols related by inversion. An alcohol and a ketone exchange two hydrogen atoms so as to produce the ketone, but with an alcohol of reverse handedness. Here $A$ and $X$ are distinct organic groups; dashes denote, in the upper panel, hydrogen bonds. The electronically excited species $G$ is postulated to be given by the structure at the bottom of the figure.

state which does not have these transformation properties. Radiatively coupling the states in the superposition then allows for the transition probabilities from $L$ and $D$ to differ, allowing for depletion of the desired enantiomer. Work is in progress to obtain qualitative insights into the essence of this symmetry breaking scheme and to design other scenarios based on a common symmetry breaking principle.

This research was supported by internal funds of the Weizmann Institute of Science and by the Natural Sciences and Engineering Research Council of Canada.

[1] R. G. Wooley, Adv. Phys. 25, 27 (1975); Origins of Optical Activity in Nature, edited by D.C. Walker (Elsevier, Amsterdam, 1979).

[2] For a discussion, see L. D. Barron, in New Developments in Molecular Chirality, edited by P. G. Mezey (Kluwer, Dordrecht, 1991); L. D. Barron, Molecular Light Scattering and Optical Activity (Cambridge University Press, Cambridge, 1982).

[3] See, for example, A. Salam and W. J. Meath, J. Chem. Phys. 106, 7865 (1997); 228, 115 (1998).

[4] Potential surfaces of this type have been discussed before [see, e.g., M. Quack, Angew. Chem. Int. Ed. Engl. 28, 571 (1989); J. A. Cina and R. A. Harris, J. Chem. Phys. 100, 2531 (1994), as well as Ref. [5]. However, none of these proposed schemes are able to lead to the enhancement of one enantiomer over another, when starting with a racemic mixture.]

[5] C. S. Maierle and R. A. Harris, J. Chem. Phys. 109, 3713 (1998).

[6] For a recent review, see M. Shapiro and P. Brumer, Adv. At. Mol. Opt. Phys. 42, 287 (2000); R. G. Gordon and S. A. Rice, Annu. Rev. Phys. Chem. 48, 595 (1997).

[7] M. Shapiro and P. Brumer, J. Chem. Phys. 95, 8658 (1991).

[8] M. Quack, Chem. Phys. Lett. 132, 147 (1986).

[9] E. Brumer, K. Baldridge, E. Deretey, M. Shapiro, and P. Brumer (to be published). 